\title{
Overexpression of transcription factor activating enhancer binding protein 4 (TFAP4) predicts poor prognosis for colorectal cancer patients
}

\author{
JIANCHANG WEI $^{1 *}$, PING YANG $^{1 *}$, TONG ZHANG $^{1 *}$, ZHUANPENG CHEN $^{1}$, \\ WEI CHEN $^{2}$, LI WANGLIN ${ }^{1}$, FENG HE ${ }^{1}$, FANG WEI ${ }^{1}$, DI HUANG ${ }^{1}$, JUNBIN ZHONG $^{1}$, \\ ZHI YANG ${ }^{1}$, HUACUI CHEN ${ }^{1}, \mathrm{HE} \mathrm{HU}^{1}$, SHANQI ZENG ${ }^{1}$, ZHENG SUN $^{1}$ and JIE CAO ${ }^{1}$ \\ ${ }^{1}$ Department of General Surgery, Guangzhou Digestive Disease Center, Guangzhou First People's \\ Hospital, Guangzhou Medical University; ${ }^{2}$ Department of Pancreatobiliary Surgery, The First \\ Affiliated Hospital, Sun Yat-sen University, Guangzhou, Guangdong 510180, P.R. China
}

Received October 3, 2016; Accepted May 15, 2017

DOI: $10.3892 / \mathrm{etm} .2017 .4875$

\begin{abstract}
Transcription factor activating enhancer binding protein 4 (TFAP4) is an important regulator in the genesis and progression of human cancers. Overexpression of TFAP4 has been found to be correlated with several malignancies. The present study assessed the clinical importance of TFAP4 in colorectal cancer (CRC). First, immunohistochemistry was used to analyze TFAP4 expression and the association of TFAP4 expression with clinicopathological features on a tissue microarray containing $208 \mathrm{CRC}$ patients. The results revealed that TFAP4 protein expression was significantly upregulated in CRC tissues compared with that in normal colon tissues $(\mathrm{P}<0.001)$. Of note, statistical analysis revealed that TFAP4 expression was significantly correlated with a high pathological grade $(\mathrm{P}=0.034)$, advanced clinical stage $(\mathrm{P}=0.024)$, enhanced tumor invasion $(\mathrm{P}=0.002)$ and lymph node metastasis $(\mathrm{P}=0.041)$. In addition, the Cancer Genome Atlas dataset further validated that TFAP4 mRNA levels were increased in CRC with advanced clinical stage $(\mathrm{P}=0.026)$, lymph node metastasis $(\mathrm{P}=0.018)$ and vascular invasion $(\mathrm{P}=0.046)$. Kaplan-Meier survival analysis demonstrated that CRC patients with high TFAP4 expression had shorter overall
\end{abstract}

Correspondence to: Professor Jie Cao, Department of General Surgery, Guangzhou Digestive Disease Center, Guangzhou First People's Hospital, Guangzhou Medical University, 1 Panfu Road, Guangzhou, Guangdong 510180, P.R. China

E-mail: czhongt@126.com

*Contributed equally

Abbreviations: TFAP4, transcription factor activating enhancer binding protein 4; CRC, colorectal cancer; TMA, tissue microarray; TCGA, the cancer genome atlas

Key words: transcription factor activating enhancer binding protein 4 , colorectal cancer, prognosis survival compared with those with low TFAP4 expression $(\mathrm{P}=0.011)$. Importantly, overexpression of TFAP4 was a valuable independent prognostic factor for CRC patients (hazard ratio, 8.200; 95\% confidence interval, 1.838-36.591; $\mathrm{P}=0.006$ ). In summary, TFAP4 may have an important role in CRC progression and upregulation of TFAP4 may be a predictor of poor prognosis for CRC patients.

\section{Introduction}

Colorectal cancer (CRC) is the second most frequently diagnosed cancer and the fourth most frequent cause for tumor-associated mortality (1). In China, it ranks 5th among all cancers in morbidity and mortality rates, with 164,900 deaths in 2013 (2). CRC remains one of the most serious health problems worldwide.

Although several biomarkers were found to be closely involved in CRC carcinogenesis and progression (3), the associated molecular mechanisms remain to be fully elucidated. Therefore, it is essential to identify novel markers, which may further clarify the biological characteristics of CRC, improve adjuvant treatments and predict clinical outcomes.

Basic helix-loop-helix (bHLH) transcription factors were identified to have important roles in cell proliferation and differentiation (4). Transcription factor activating enhancer binding protein 4 (TFAP4) is a member of the bHLH leucine-zipper subgroup (5), which was first reported in 1988 (6). Studies revealed that TFAP4 was overexpressed in gastric cancer (7), hepatocellular carcinoma (8) and non-small cell lung carcinoma (9), and overexpression of TFAP4 predicted poor prognosis. Cao et al (10) found that high expression of TFAP4 was correlated with poor differentiation, advanced Dukes stage and shorter 5-year survival. In addition, TFAP4 was found to be a mediator of epithelial-mesenchymal transition and metastasis in CRC (11). However, the prognostic significance of TFAP4 in CRC has remained to be fully elucidated.

In the present study, a CRC tissue microarray (TMA) and The Cancer Genome Atlas (TCGA) dataset were used to investigate the expression of TFAP4 in CRC and its association 
with the clinicopathological characteristics and prognosis of patients with CRC.

\section{Materials and methods}

Tissue samples. TCGA (https://tcga-data.nci.nih.gov/docs/ publications/tcga/) is a public dataset with clinical information and mRNA expression of all patients (12). The TMA (http://www.alenabio.com/CO2161; no. CO2161) with 208 CRC and eight normal colon tissues was obtained from US Biomax (Rockville, MD, USA), accompanied with clinical information of all patients.

Immunohistochemistry (IHC). The Dako EnVision System (cat. no. K4004; Agilent Technologies, Inc., Santa Clara, CA, USA) was used for the IHC assay according to the manufacturer's instructions. In brief, after blockage of TMA specimens by proteolytic digestion and peroxidase, the slides were incubated overnight with rabbit anti-human TFAP4 polyclonal antibody (cat. no. ab28512; 1:50 dilution; Abcam, Cambridge, UK) at $4^{\circ} \mathrm{C}$. The slides were washed with PBS and then incubated with horseradish peroxidase-labeled antibodies (cat. no. K4004; 1:100 dilution; Agilent Technologies, Inc.) for $30 \mathrm{~min}$ at room temperature. Substrate chromogen (cat. no. K4004; Agilent Technologies, Inc.) was added for $1 \mathrm{~min}$ to visualize protein staining.

Evaluation of immunostaining. The TMA was analyzed using ImageScope v11 software (Aperio Technologies, Inc., Vista, CA, USA). Two pathologists were blinded to patient information and evaluated protein expression in a semi-quantitative manner. Scoring for staining intensity in each specimen was performed as follows: 0 , no staining; 1 , weak; 2 , moderate; and 3 , strong. The percentage of staining was scored as follows: $1,0-25 ; 2,25-50,3,50-75$ and 4, 75-100\%. The immunoreactivity score (IRS) was determined by the addition of intensity and percentage scores. The scores given by the pathologists were compared and any discrepant scores were reevaluated to achieve a consensus score. Samples were divided into those with high (IRS>4) and low (IRS $\leq 4$ ) TFAP4 levels (13).

Statistical analysis. SPSS 21.0 software (International Business Machines, Corp., Armonk, NY, USA) was used for statistical analyses. The correlation between TFAP4 and clinicopathological characteristics was analyzed by Fisher's exact test and Pearson's $\chi^{2}$ test. Overall survival was analyzed using the Kaplan-Meier method and the log-rank test. Cox proportional hazards regression was performed for univariate and multivariate survival analyses. The relative risk of death was expressed as the adjusted hazard ratio (HR) with the corresponding $95 \%$ confidence interval $(\mathrm{CI}) . \mathrm{P}<0.05$ was considered to indicate a statistically significant difference.

\section{Results}

TFAP4 is significantly upregulated in CRC tissues. In order to explore the expression of TFAP4 in CRC, the present study first detected TFAP4 expression in a TMA by IHC (Fig. 1A). TFAP4 levels in CRC tissues were higher compared with those in normal colon tissues (IRS: $6.89 \pm 2.52$ vs. $3.25 \pm 1.04$;
$\mathrm{P}<0.001$; Fig. 1B). As presented in Fig. 1C and D, TFAP4 signals were detected on the plasma membrane and in the cytoplasm of CRC cells. Of the 204 CRC samples, 46 (22.5\%) had low TFAP4 expression, while 158 (77.5\%) had high expression.

The present study further evaluated whether TFAP4 expression was associated with clinicopathological characteristics of CRC patients in the TMA cohort. As presented in Table I, overexpression of TFAP4 was associated with a high pathological grade $(\mathrm{P}=0.034)$, advanced clinical stage $(\mathrm{P}=0.024)$, enhanced tumor invasion $(\mathrm{P}=0.002)$ and lymph node metastasis $(\mathrm{P}=0.041)$. These findings indicated that TFAP4 may have an important role in CRC progression.

High TFAP4 mRNA levels are correlated with CRC progression and poor prognosis. To validate the findings of the TMA, the TCGA dataset comprising 192 primary CRC tissues and 51 normal colorectal tissues was analyzed. It was found that TFAP4 mRNA was significantly upregulated in CRC tissues compared with that in normal tissues $(\mathrm{P}<0.001$; Fig. 2A). The correlation of TCGA mRNA expression with clinicopathological characteristics was then analyzed in the 192 CRC patients. As presented in Table I, TFAP4 mRNA was significantly upregulated in $\mathrm{CRC}$ with advanced clinical stage $(\mathrm{P}=0.026)$, lymph node metastasis $(\mathrm{P}=0.018)$ and vascular invasion $(\mathrm{P}=0.046)$.

To further evaluate the prognostic value of TFAP4 in CRC patients, Kaplan-Meier survival curves were drawn to compare the prognosis between patients with high and low TFAP4 expression from the TCGA dataset (Fig. 2B). The mean TFAP4 mRNA level (556.59 \pm 208.47 counts of fragments per kilobase million) in the TCGA dataset was used as a cutoff value to divide patients into high and low TFAP4 expression groups (13). Overall survival time after surgery in the high and low TFAP4 expression groups were 39.41 \pm 5.372 and $45.40 \pm 3.124$ months, respectively. Importantly, patients with higher TFAP4 expression had a shorter survival time, whereas patients with lower TFAP4 expression had a longer overall survival time (log-rank, 6.507; $\mathrm{P}=0.011)$.

TFAP4 is an independent prognostic factor for the survival of CRC patients. Finally, the Cox proportional hazards model was used to evaluate whether TFAP4 is a valuable predictor for the survival of CRC patients from the TCGA dataset. As presented in Table II, univariate analysis indicated that TFAP4 was a significant prognostic factor (HR, 3.332; 95\% CI, 1.245-8.923; $\mathrm{P}=0.017)$, and multivariate analysis further revealed that TFAP4 was an independent prognostic factor in CRC (HR, 8.200; 95\% CI, 1.838-36.591; P=0.006).

\section{Discussion}

TFAP4 is a transcription factor that is ubiquitously expressed in human tissues and recognizes the E-box sequence CAGCTG in the promoters of target proteins (14). A previous study demonstrated that the TFAP4 gene is a direct transcriptional target of c-MYC, and inhibited cell cycle arrest induced by DNA damage and differentiation (15). Another study reported that MYCN promoted the malignancy of neuroblastoma by regulating the expression of TFAP4 (16). 
Table I. Correlation of transcription factor activating enhancer binding protein 4 expression with clinicopathological characteristics of colorectal cancer patients.

\begin{tabular}{|c|c|c|c|c|c|c|c|}
\hline \multirow[b]{2}{*}{ Clinical feature } & \multicolumn{4}{|c|}{ TMA } & \multicolumn{3}{|c|}{ TCGA } \\
\hline & Total (n) & Low n $(\%)$ & High n (\%) & $\begin{array}{c}\text { P-value } \\
\left(\chi^{2} \text { value }\right)\end{array}$ & Total (n) & $\begin{array}{c}\text { TFAP4 levels } \\
\text { (mean } \pm \text { standard } \\
\text { deviation) }\end{array}$ & P-value \\
\hline Age & & & & $0.955(0.003)$ & & & 0.735 \\
\hline$<60$ & 119 & $27(22.7)$ & $92(77.3)$ & & 38 & $566.90 \pm 236.07$ & \\
\hline$\geq 60$ & 85 & $19(22.4)$ & $66(77.6)$ & & 154 & $554.05 \pm 201.85$ & \\
\hline Gender & & & & $0.834(0.044)$ & & & 0.958 \\
\hline Male & 117 & $27(23.1)$ & $90(76.9)$ & & 94 & $557.41 \pm 200.82$ & \\
\hline Female & 87 & $19(21.8)$ & $68(78.2)$ & & 98 & $555.81 \pm 216.58$ & \\
\hline Pathological grade & & & & $0.034(4.543)$ & & & \\
\hline$\leq 2$ & 146 & $37(25.3)$ & $109(74.7)$ & & - & - & - \\
\hline$>2$ & 41 & $4(9.8)$ & $37(90.2)$ & & - & - & - \\
\hline Clinical stage & & & & $0.024(5.097)$ & & & 0.026 \\
\hline I-II & 146 & $39(26.7)$ & $107(73.3)$ & & 108 & $527.62 \pm 204.84$ & \\
\hline III-IV & 58 & $7(12.1)$ & $51(87.9)$ & & 81 & $595.60 \pm 209.22$ & \\
\hline Tumor invasion & & & & $0.002(9.484)$ & & & 0.586 \\
\hline $\mathrm{T} 1-\mathrm{T} 2$ & 23 & $11(47.8)$ & $12(52.2)$ & & 44 & $543.02 \pm 256.64$ & \\
\hline T3-T4 & 181 & $35(19.3)$ & $146(80.7)$ & & 132 & $562.84 \pm 190.00$ & \\
\hline Lymph node metastasis & & & & $0.041(4.159)$ & & & 0.018 \\
\hline No & 149 & $39(26.2)$ & $110(73.8)$ & & 114 & $526.22 \pm 202.95$ & \\
\hline $\mathrm{N} 1-2$ & 55 & $7(12.7)$ & $48(87.3)$ & & 77 & $598.73 \pm 210.17$ & \\
\hline Distant metastasis & & & & $0.462(1.206)$ & & & 0.760 \\
\hline M0 & 193 & $45(23.3)$ & $148(76.7)$ & & 158 & $555.94 \pm 212.82$ & \\
\hline M1 & 11 & $1(9.1)$ & $10(90.9)$ & & 30 & $568.68 \pm 185.61$ & \\
\hline Vascular invasion & & & & & & & 0.046 \\
\hline No & - & - & - & - & 118 & $536.81 \pm 212.20$ & \\
\hline Yes & - & - & - & - & 41 & $613.62 \pm 206.31$ & \\
\hline
\end{tabular}

'-' indicated a lack of related information for the patient. TMA, tissue microarray; TCGA, the cancer genome atlas.

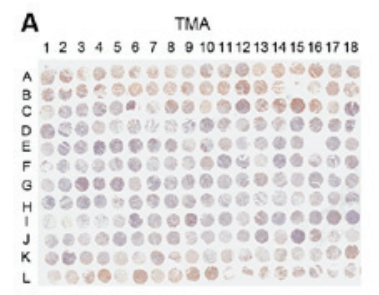

B

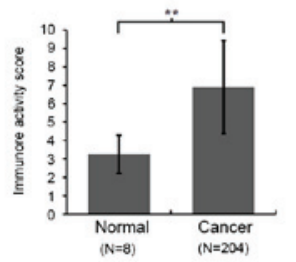

D

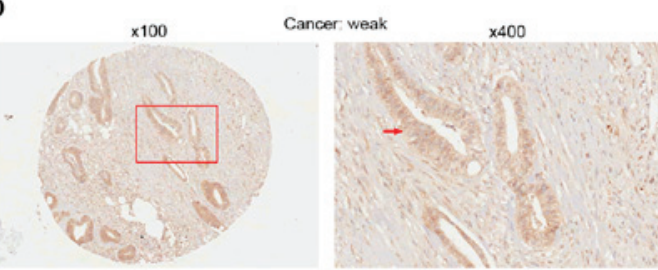

C

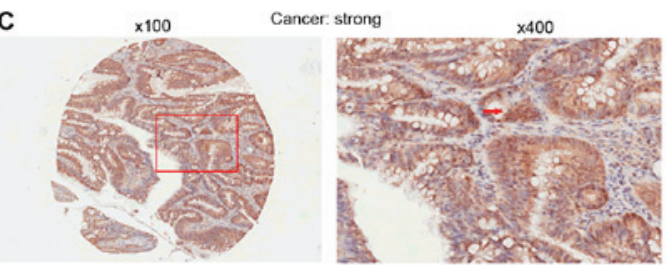

E

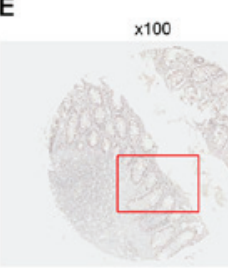

Normal: negative

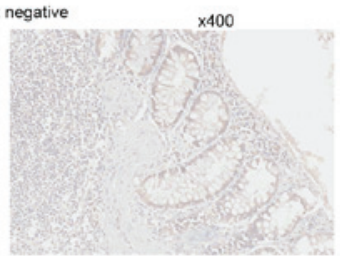

Figure 1. Immunohistochemical staining for TFAP4 in colon cancer and normal colon tissues. (A) Staining pattern of the TMA. (B) Immunoreactive score in cancerous tissues were higher than those in normal colon tissues. ${ }^{* *} \mathrm{P}<0.01$. (C) Microscopy image of sample B12 in A and an enlarged window, demonstrating a strong staining for TFAP4 on the plasma membrane and in the cytoplasm of cancer cells. (D) Microscopy image of sample A13 in A and an enlarged window, demonstrating that TFAP4 expression was weak in the cytoplasm of cancer cells. (E) Microscopy image of sample I16 in A and an enlarged window, revealing that TFAP4 expression was negative in the normal colon tissues. The red arrows indicate positively stained cells (original magnifications, x100 and x400). TFAP4, transcription factor activating enhancer binding protein 4; TMA, tissue microarray. 
Table II. Prognostic value of TFAP4 expression regarding overall survival in the Cancer Genome Atlas dataset.

\begin{tabular}{|c|c|c|c|c|}
\hline \multirow[b]{2}{*}{ Variable } & \multicolumn{2}{|c|}{ Univariate analysis } & \multicolumn{2}{|c|}{ Multivariate analysis } \\
\hline & $\mathrm{HR}(95 \% \mathrm{CI})$ & P-value & $\mathrm{HR}(95 \% \mathrm{CI})$ & P-value \\
\hline Clinical stage (III/IV vs. I/II) & $1.809(0.735-4.453)$ & 0.197 & $1.027(0.081-13.006)$ & 0.984 \\
\hline $\mathrm{N}$ stage (N1/2 vs. N0) & $1.633(0.674-3.956)$ & 0.278 & $0.824(0.089-7.613)$ & 0.865 \\
\hline M stage (M1 vs. M0) & $2.706(1.023-7.162)$ & 0.045 & $1.890(0.457-7.813)$ & 0.379 \\
\hline Vascular invasion (yes vs. no) & $5.089(1.704-15.197)$ & 0.004 & $2.990(0.986-9.064)$ & 0.053 \\
\hline TFAP4 expression (high vs. low) & $3.332(1.245-8.923)$ & 0.017 & $8.200(1.838-36.591)$ & 0.006 \\
\hline
\end{tabular}

HR, hazard ratio; CI, confidence interval; TFAP4, transcription factor activating enhancer binding protein 4.

A

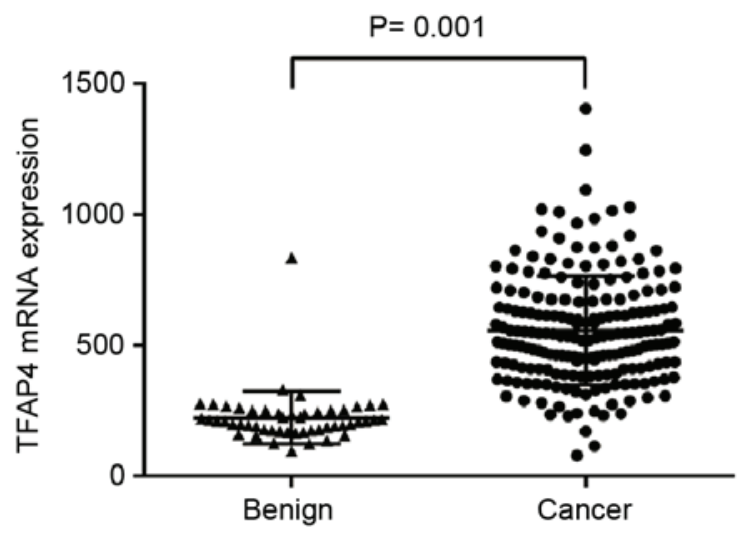

B

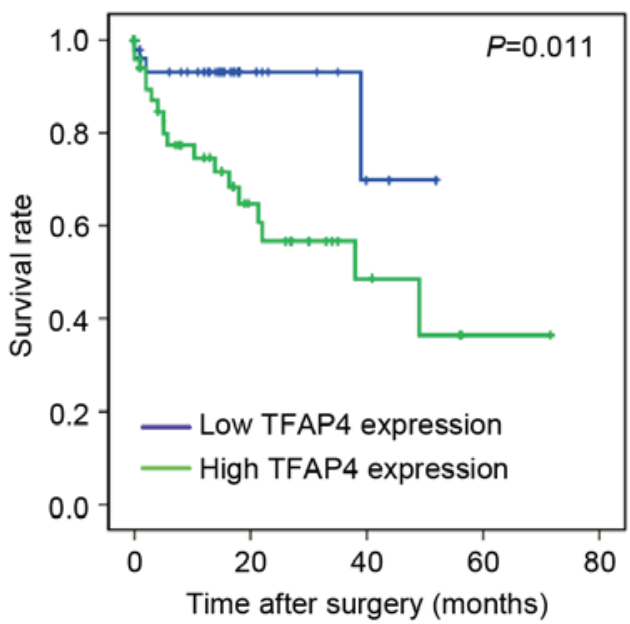

Figure 2. Clinical association of TFAP4 with CRC according to the data from the Cancer Genome Atlas. (A) Expression of TFAP4 in CRC and normal tissues. Data were presented as the mean \pm standard deviation. (B) Overall survival of CRC patients with high and low TFAP4. TFAP4, transcription factor activating enhancer binding protein 4 ; CRC, colorectal cancer.

A recent study reported that silencing of TFAP4 inhibited human lung cancer cell proliferation, caused cell cycle arrest at G0/G1 phase and induced apoptosis by modulating p21 and cyclin D1, suggesting that TFAP4 may be an oncoprotein and may have therapeutic potential in lung cancer (17). Liu et al (18) also found that knockdown of TFAP4 inhibited gastric cancer cell proliferation, induced apoptosis and sensitized cancer cells to anti-cancer drugs, indicating that TFAP4 has a potential drug target value in the treatment of gastric cancer.

TFAP4 was also identified as a prognostic factor in several cancer types, including gastric cancer (7), hepatocellular carcinoma (8) and non-small cell lung cancer (9). However, the prognostic value of TFAP4 in CRC has remained elusive. To investigate this, the present study assessed TFAP4 protein levels in a TMA containing 193 CRC and 8 normal colon tissues. IHC analysis revealed that TFAP4 was located on the plasma membrane and in the cytoplasm of CRC cells, and TFAP4 was upregulated in CRC compared with normal tissues. In addition, overexpression of TFAP4 was found to be significantly associated with a high pathological grade, advanced clinical stage, enhanced tumor invasion and lymph node metastasis (all $\mathrm{P}<0.05$ ). More importantly, these findings were further validated in the TCGA dataset, where TFAP4 upregulation was significantly associated with advanced clinical stage, lymph node metastasis and vascular invasion (all $\mathrm{P}<0.05$ ). Taken together, these findings indicated that TFAP4 may have an oncogenic role in the carcinogenesis and progression of CRC.

Furthermore, overall survival was compared to assess the prognostic value of TFAP4 expression in CRC patients from the TCGA dataset. It was found that overexpression of TFAP4 was significantly correlated with shorter overall survival. The Cox proportional hazards model further revealed that TFAP4 was an independent predictive factor for the survival of CRC patients.

In conclusion, although the findings of the present study require further validation by analysis of additional samples and the exact molecular function of TFAP4 remains to be clarified, the present study suggested that TFAP4 has an important role in CRC progression and may be a valuable and independent prognostic factor for CRC patients.

\section{Acknowledgements}

The study was supported by grants from the National Natural Science Foundation of China (grant no. 81272556), 
the Science and Technology Project of Guangdong Province (grant no. 2014A020212614) and the Science and Technology Program of Guangzhou, China (grant no. 2014Y2-00137).

\section{References}

1. Torre LA, Bray F, Siegel RL, Ferlay J, Lortet-Tieulent J and Jemal A: Global cancer statistics, 2012. CA Cancer J Clin 65: 87-108, 2015.

2. Chen W, Zheng R, Zhang S, Zeng H, Xia C, Zuo T, Yang Z, Zou X and He J: Cancer incidence and mortality in China, 2013. Cancer Lett 401: 63-71, 2017.

3. Li X, Liang L, Huang L, Ma X, Li D and Cai S: High expression of protein phosphatase 4 is associated with the aggressive malignant behavior of colorectal carcinoma. Mol Cancer 14: 95 , 2015 .

4. Sakar Y, Duca FA, Langelier B, Devime F, Blottiere H, Delorme C, Renault P and Covasa M: Impact of high-fat feeding on basic helix-loop-helix transcription factors controlling enteroendocrine cell differentiation. Int J Obes (Lond) 38: 1440-1448, 2014.

5. Lee SU, Song HO, Lee W, Singaravelu G, Yu JR and Park WY: Identification and characterization of a putative basic helix-loop-helix (bHLH) transcription factor interacting with calcineurin in C. elegans. Mol Cells 28: 455-461, 2009.

6. Mermod N, Williams TJ and Tjian R: Enhancer binding factors AP-4 and AP-1 act in concert to activate SV40 late transcription in vitro. Nature 332: 557-561, 1988.

7. Xinghua L, Bo Z, Yan G, Lei W, Changyao W, Qi L, Lin Y, Kaixiong T, Guobin W and Jianying C: The overexpression of AP-4 as a prognostic indicator for gastric carcinoma. Med Oncol 29: 871-877, 2012.

8. Hu BS, Zhao G, Yu HF, Chen K, Dong JH and Tan JW: High expression of AP-4 predicts poor prognosis for hepatocellular carcinoma after curative hepatectomy. Tumour Biol 34: 271-276, 2013.
9. Gong H, Han S, Yao H, Zhao H and Wang Y: AP-4 predicts poor prognosis in non-small cell lung cancer. Mol Med Rep 10: 336-340, 2014

10. Cao J, Tang MH, Li WL, Xie J, Du H, Tang WB, Wang H, Chen XW, Xiao H and Li Y: Upregulation of activator protein-4 in human colorectal cancer with metastasis. Int J Surg Pathol 17: 16-21, 2009.

11. Jackstadt R, Röh S, Neumann J, Jung P, Hoffmann R, Horst D, Berens C, Bornkamm GW, Kirchner T, Menssen A and Hermeking H: AP4 is a mediator of epithelial-mesenchymal transition and metastasis in colorectal cancer. J Exp Med 210: 1331-1350, 2013.

12. The Cancer Genome Atlas Network: Comprehensive molecular characterization of human colon and rectal cancer. Nature 487: 330-337, 2012.

13. Yang P, Wei J, Li W, He F, Zeng S, Zhang T, Sun Z and Cao J: High expression of growth factor receptor-bound protein 14 predicts poor prognosis for colorectal cancer patients. Biotechnol Lett 38: 1043-1047, 2016.

14. Hu YF, Lüscher B, Admon A, Mermod N and Tjian R: Transcription factor AP-4 contains multiple dimerization domains that regulate dimer specificity. Genes Dev 4: 1741-1752, 1990.

15. Jung P, Menssen A, Mayr D and Hermeking H: AP4 encodes a c-MYC-inducible repressor of p21. Proc Natl Acad Sci 105: 15046-15051, 2008.

16. Xue C, Yu DM, Gherardi S, Koach J, Milazzo G, Gamble L, Liu B, Valli E, Russell AJ, London WB, et al: MYCN promotes neuroblastoma malignancy by establishing a regulatory circuit with transcription factor AP4. Oncotarget 7: 54937-54951, 2016.

17. Hu X, Guo W, Chen S, Xu Y, Li P, Wang H, Chu H, Li J, DU Y, Chen X, et al: Silencing of AP-4 inhibits proliferation, induces cell cycle arrest and promotes apoptosis in human lung cancer cells. Oncol Lett 11: 3735-3742, 2016.

18. Liu X, Zhang B, Guo Y, Liang Q, Wu CY, Wu L, Tao K, Wang G and Chen J: Down-regulation of AP-4 inhibits proliferation, induces cell cycle arrest and promotes apoptosis in human gastric cancer cells. PLoS One 7: e37096, 2012. 